\title{
STEUERN UND STEUERERHEBUNG IN COSTA RICA UND GUATEMALA ${ }^{1)}$
}

Von Hermann Avenarius und Jürgen H. WolfF

\section{A. COSTA RICA}

\section{Einführung}

Innerhalb der mittelamerikanischen Staatengruppe zeichnet sich Costa Rica durch geringe soziale Spannungen und eine verhältnismäßig ausgeglichene Gesellschaftsstruktur aus. Die Zuwanderung vorwiegend bäuerlicher, vor allem aus Nordspanien stammender Bevölkerungsgruppen hat die Herausbildung einer relativ homogenen Struktur landwirtschaftlichen Kleinbesitzes gefördert und das Entstehen einer „Hacienda-Gesellschaft" verhindert. Ein extremer Dualismus Latifundium - Minifundium, wie er in den Nachbarländern besteht, blieb Costa Rica erspart.

Mit der Abschaffung des Militärs 1948 freiwerdende Mittel erlaubten den gezielten Ausbau des allgemeinbildenden Primar- und Sekundarschulwesens. Seit Jahren werden über $25 \%$ der Staatsausgaben für Erziehungs- und Ausbildungszwecke verwendet. Mit nur etwa $13 \%$ Analphabeten unterbietet Costa Rica die meisten lateinamerikanischen Länder. Man kann heute sogar davon sprechen, daß die Zahl der ausgebildeten Arbeitssuchenden größer als die der verfügbaren Arbeitsplätze ist.

Trotz des hohen sozialen und kulturellen Entwicklungsstandes ist die ökonomische Struktur keineswegs zufriedenstellend. Zwar gehört das Land hinsichtlich seines Pro-Kopf-Einkommens von weit mehr als US-\$500,- zu den fünf führenden Ländern Lateinamerikas, doch ist die Wirtschaft in hohem Grade von wenigen für den Export produzierten landwirtschaftlichen Gütern abhängig. Kaffee und Bananen machen zusammen $56 \%$ des Exporterlöses aus. Der überwiegende Teil der Bevölkerung ist in der Landwirtschaft tätig. Das Land ist regional sehr ungleich erschlossen. Die Zentralzone ist stark urbanisiert; allein in Groß-San José leben etwa $25 \%$ der Bevölkerung. In den Randzonen sind große Landstriche unbesiedelt und nicht bebaut.

Der politische Prozeß in der gut funktionierenden parlamentarischen Demokratie stützt sich auf eine aktive Partizipation breiter Bevölkerungsschichten. Der bürgerkriegsähnliche Konflikt des Jahres 1948 brachte die Rückkehr zum Verfassungsstaat und ermöglichte den Aufbau eines sozial orientierten demokratischen Gemeinwesens. Seither haben sich die wichtigsten Parteien fast regelmäßig in der Regierungsverantwortung abgewechselt. Darin schlagen sich ein waches demokratisches Bewußtsein und eine beachtliche politische Dynamik der Wählerschaft nieder.

Die regional ungleiche Erschließung des Landes kommt auch in der starken Konzentration der staatlichen Verwaltung auf die dicht besiedelte metropolitane Zone

\footnotetext{
1 Der Aufsatz beruht auf Ergebnissen einer Studie, welche die Verfasser gemeinsam mit Dieter Oberndörfer und Ulrich Fanger im Auftrag des Bundesministeriums für wirtschaftliche Zusammenarbeit 1973 erstellt haben. Diese Untersuchung behandelt neben den im vorliegenden Beitrag dargestellten Problemen das Personalwesen der Steuerverwaltung und Fragen der staatlichen Finanzkontrolle.
} 
zum Ausdruck. Sie läßt den sieben Provinzen - mit ihren Untereinheiten: den Kantonen und Distrikten - nur beschränkte Befugnisse und benachteiligt die Bevölkerung der Randprovinzen auch in ihrem Anteil am öffentlichen Dienst. Andererseits haben die festverankerten demokratischen Traditionen den Ausbau einer von der Exekutive weitgehend unabhängigen, korruptionsfreien und zunehmend an objektiven Leistungskriterien orientierten Administration ermöglicht.

\section{Das Steuersystem}

Das Steuersystem Costa Ricas ist kompliziert und in den Einnahmearten sehr ungleichwertig. Von insgesamt 62 Einzelpositionen des Haushaltsplans $1973^{2}$ erbrachten die 41 fiskalisch unbedeutendsten nur 5,3 Prozent der staatlichen Einnahmen. Demgegenüber betrug das Aufkommen aus den vier wichtigsten Steuern (Einkommen-, Verbrauchs- und Umsatzsteuer sowie Importzöllen) 64,4 Prozent des gesamten Budgets. Die Untersuchung beschränkt sich auf diese Abgaben; sie bezieht im übrigen die unter sozialpolitischen Gesichtspunkten potentiell wichtige Grundsteuer ein.

Der Einkommensteuer ${ }^{3}$ sind natürliche Personen und Kapitalgesellschaften mit unterschiedlichen Steuersätzen unterworfen. Der von einem Unternehmen ausgeschüttete Gewinn ist zusätzlich beim Anteilseigner zu versteuern. Die Steuersätze für natürliche Personen sind relativ hoch und weisen eine starke Progression auf; sie setzen bei einem zu versteuernden Jahreseinkommen bis zu C $5000^{4} \mathrm{mit}$ 5 Prozent ein und erreichen bereits bei einem Betrag von C 350000 die obere Proportionalzone von 50 Prozent. Demgegenüber werden die Erträge der Körperschaften maßvoll besteuert; die Eingangsstufe von 5 Prozent erfaßt Gewinne bis zu C 50 000, der Höchsttarif von 40 Prozent fällt erst bei einer Summe von C 1000000 an. Sachlich nicht gerechtfertigte Vergünstigungen, etwa überhöhte Freibeträge für privilegierte Berufsgruppen, sind nicht vorgesehen.

Die Einkommensteuer hat in den vergangenen Jahren stark an Bedeutung gewonnen. Die aus ihr erzielten Einnahmen betrugen 1966 C 82,37 Mio. und beliefen sich nach dem Haushaltsvoranschlag für 1973 auf C 195,0 Mio. Ihr Anteil an den gesamten Steuereinnahmen stieg von 17,5 Prozent (1966) auf 21,7 Prozent (1971) ${ }^{5}$.

Der Verbrauchssteuer ${ }^{6}$ unterliegt der Konsum einer Vielzahl von Gütern. Insbesondere Luxusgegenstände werden mit hohen Abgaben (bis zu 50 Prozent) belegt. Die 1972 neu geltenden relativ hohen Steuersätze haben zu einer erheblichen Zu* nahme des Aufkommens geführt: von C 39,4 Mio. im Jahre 1971 auf C 125,0 Mio. im Jahre 1972. 1973 wurden sogar Erträge von C 183,0 Mio. erwartet ${ }^{7}$. Damit hat

2 Contraloría General de la República, Departamento de Estudios Económicos: Presupuesto de Ingresos, Tipo Programas 1973, San José 1972.

3 Die folgende Darstellung stützt sich, soweit nichts anderes vermerkt, auf die folgenden statistischen Werke:

a) República de Costa Rica, Ministerio de Hacienda, Dirección, General de la Tributación Directa: Estadística Demográfica Fiscal del Impuesto sobre la Renta, Periodo 39, San José 1972;

b) Contraloría General de la República: Presupuesto-Tipo Programas, a. a. O.; sowie auf den Wortlaut des Gesetzes:

c) Impuesto sobre la Renta, Ley No. 837 vom 20. 12. 1946; vielfach novelliert, zuletzt durch Gesetz No. 4961 v. 10. 3. 72, abgedruckt in der Loseblatt-Gesetzessammlung "Legislación al Día ${ }^{\alpha}$.

4 Kurs 1973: 1 US- $\$=C$ (Colones) 8,60.

5 Zahlen nach Contraloría General: Presupuesto-Tipo Programas, a. a. O. sowie dem Haushaltsplan 1973.

6 Rechtsgrundlage ist Gesetz Nr. 4961 a. d. J. 1972.

7 Quelle: Contraloría General: Presupuesto-Tipo Programas, a. a. O. und Haushaltsplan 1973. 
sich der Anteil der Verbrauchssteuer an den gesamten staatlichen Steuereinnahmen von 4,7 Prozent auf 23,3 Prozent erhöht.

Die Umsatzsteuer ${ }^{8}$ erfaßt als Einphasensteuer den Verkauf von Gütern und Dienstleistungen auf der Stufe des Endabnehmers mit einem einheitlichen Steuersatz von 5 Prozent. Aus sozialen Erwägungen und zur Vermeidung von Doppelbelastungen (vor allem zusätzlich zur Verbrauchssteuer) sind viele Erzeugnisse freigestellt. Die Erträge aus der Umsatzsteuer wuchsen von C 74,87 Mio. ${ }^{9}$ (1968) auf 140,0 Mio. (1973) ${ }^{10}$. Ihr Anteil am gesamten Steueraufkommen ist jedoch in den vergangenen Jahren mit etwas mehr als 13 Prozent gleich geblieben.

Die Importzölle ${ }^{11}$ verlieren im Zuge der zentralamerikanischen Wirtschaftsintegration mehr und mehr an fiskalischer Bedeutung. Zur Förderung des zwischenstaatlichen Handels sind die meisten Einfuhrgüter aus den Mitgliedsländern des Gemeinsamen Marktes von Importbelastungen befreit worden. Nicht zuletzt deshalb ist der Anteil der Importzölle an den staatlichen Steuereinnahmen erheblich zurückgegangen: von 46,68 Prozent (1963) auf 22,81 Prozent (1971) ${ }^{12}$. Der Anteil am Importwert betrug im 2. Quartal 1972 7,23 Prozent; es sei allerdings nochmals betont, daß es sich bei diesem relativ niedrigen Wert nicht um die gesamte Einfuhrbelastung handelt.

Die Grundsteuer ${ }^{13}$ erfaßt Grundstücke mit einem Wert von mehr als C 10000. Sie ist mit einem progressiven Tarif ausgestattet, der je nach Wert der Liegenschaft zwischen 0,3 Prozent und 1,05 Prozent beträgt. Die Erträge aus dieser Abgabe fließen zum größten Teil den Kommunen $\mathrm{zu}^{14}$. Der mit der Einführung der Steuer verfolgte $Z_{w e c k}$, den Großgrundbesitz einzuschränken - der allerdings eine weit geringere Bedeutung als in anderen lateinamerikanischen Staaten aufweist und auf diesem Weg die Agrarreform zu fördern, ist nicht erreicht worden. Die Steuersätze sind zu niedrig. Vielfach werden Grund und Boden weit unter ihrem tatsächlichen Wert deklariert. Ob die ins Auge gefaßte Reform des Grundsteuergesetzes die gegenwärtigen Mängel zu überwinden vermag, bleibt abzuwarten. Immerhin ist das Aufkommen aus der Grundsteuer in den letzten Jahren von C 24,05 Mio. (1966) auf C 40,92 Mio. (1971) gestiegen ${ }^{15}$; der Anteil an den gesamten Steuereinnahmen hat sich demgegenüber mit 5,10 Prozent (1971) gegenüber 4,64 Prozent (1966) kaum verändert.

Die (Zusatz-)Steuer auf unbebautes Land ${ }^{16}$, deren Erträge für das Landreforminstitut ITCO bestimmt sind, steht nur auf dem Papier. Den Großgrundbesitzern ist es bislang gelungen, sich dieser Abgabe zu entziehen.

\footnotetext{
8 Die Darstellung folgt dem 1967 eingeführten und bis heute gültigen Gesetz Nr. 3914 sowie dem Reglarnento de la Ley sobre las Ventas, Decreto Ejecutivo Nr. 2381 von 1972.

9 Contraloría General: Presupuesto-Tipo Programas, a. a. O., S. 20.

10 Nach dem Haushaltsvoranschlag.

11 Es handelt sich nicht um die einzige Belastung importierter Waren. Neben den verschiedenen Umsatzund Verbrauchssteuern, die auch beim Import fällig werden, sind die Abgaben gemäß dem „Protokoll von San José" von 1970 (zur Zahlungsbilanzstabilisierung der Mitgliedsländer des Gemeinsamen Marktes) sowie die unter der Bezeichnung "Impuesto Interior y Otras Rentas Aduaneras “ zusammengefaßten Abgaben zu nennen, die im Haushalt $1973 \mathrm{mit} C 60,05$ Mio. veranschlagt waren (s. Presupuesto-Tipo Programas, a. a. O., Tab. vor S. 1, S. 22, 24). Zum folgenden siehe: David Gantz: La aplicación de la Ley de Protección y Desarrollo Industrial (Costa Rica), in: Revista de Ciencias Jurídicas (Universidad de Costa Rica), No. 14, Dez. 1969, S. 113-284; Edgardo Paz Barnica: Reestructuración institucional de la integración Centroamericana, o. O. (Tegucigalpa?) 1972; Rolando Soto Jiménez: Los problemas tributarios de los paises Centroamericanos y su relación con el proceso de la integración. El caso de tributarios de los paises Centroamericanos y su relacion con el proceso de la integración. El caso de
los impuestos aduaneros, in: Revista de Ciencias Jurídicas (Univ. de C. R.), No. 16, Nov. 1970, S. $199-231$.

12 Quellen wie oben.

13 Rechtsgrundlagen sind heute Gesetz Nr. 27 (von 1939) und 4340 (von 1969).

14 Dieses war der wesentliche Inhalt des zit. Gesetzes 4340.

15 Quelle: Dirección General de la Tributación Directa: Memoria anual 1972, S. 16.

16 Impuesto sobre Tierras Incultas, Gesetz 3042 von 1962.
} 
Zum Steuersystem insgesamt ist folgendes zu bemerken:

1. Die indirekte Besteuerung übersteigt die direkte bei weitem. Letztere erbrachte nach den Haushaltsansätzen für 1973 nur 21,5 Prozent der Steuer- und 21,3 Prozent der Gesamteinnahmen des Staates ${ }^{17}$. Indirekte Steuern und sonstige regelmäßige Einnahmen, insbesondere Gebühren, trugen also 1973 die Staatsfinanzen zu 78,7 Prozent ${ }^{18}$.

Was den Anteil der direkten Steuern am gesamten staatlichen Steueraufkommen angeht, so nimmt Costa Rica im Vergleich zu anderen lateinamerikanischen Ländern eine mittlere Position ein ${ }^{19}$.

Folgt man der klassischen finanzwissenschaftlichen Theorie, die zwischen direkten und indirekten Steuern nach dem Kriterium der Überwälzbarkeit unterscheidet, so erweist sich das Steuersystem Costa Ricas als regressiv oder doch nur geringfügig progressiv: die Bezieher höherer Einkommen werden wegen des Vorrangs der überwiegend nicht an der persönlichen wirtschaftlichen Leistungsfähigkeit orientierten indirekten Steuern relativ weniger stark als die ärmeren Bevölkerungsschichten belastet. Die gegenwärtige Fiskalpolitik verstößt danach gegen das Gebot steuerlicher Gerechtigkeit. Ob sich dieses Urteil auch dann noch aufrechterhalten läßt, wenn man mit verschiedenen Autoren ${ }^{20}$ davon ausgeht, daß bei Vorliegen bestimmter gesamtwirtschaftlicher Daten und eines bestimmten Verhaltens der Wirtschaftssubjekte grundsätzlich alle Steuern überwälzt werden können, ist ungewiß. Die wissenschaftliche Diskussion führte jedenfals nicht zu Maßkonzepten der Überwälzung im konkreten Fall21.

2. Das Steuersystem ist kompliziert, dabei im Aufkommen äußerst ungleichgewichtig. Diese Tatsache legt eine radikale Vereinfachung des Systems nahe; im Hinblick auf die geringen Erträge der meisten Steuern erscheint eine Reform nicht allzu schwierig.

3. Das grundlegende Budgetierungsprinzip der Einheit der Staatsfinanzen (d. h.: alle Einnahmen sind in eine einzige Kasse überzuführen, alle Ausgaben sind aus dieser zu begleichen) wird in zahlreichen Fällen nicht beachtet ${ }^{22}$. So werden vor allem die kleinen speziellen Verbrauchssteuern von vornherein bestimmten Organisationen zugewiesen. Besonders die autonomen und halbautonomen Körperschaften bzw. Organisationen haben mit dem aus der Sicht des Behördenchefs verständlichen Bestreben Erfolg gehabt, ihre Sonderfinanzierung langfristig unabhängig vom Bewilligungsrecht des Parlaments zu sichern.

Ahnliche Folgen hat in diesem Zusammenhang die Tatsache, daß Anteile des Staatshaushaltes von vornherein bestimmten Verwaltungszweigen zufließen. Hier liegt also nicht die Zweckbindung einer Einzelsteuer, sondern vielmehr von Teilen der Gesamtausgaben vor. Das gilt etwa für die Justiz (Art. 177, Abs. 2 der Verfassung),

17 Werden die regelmäßigen Gemeindeeinkünfte einbezogen, dann verändert sich das Bild allerdings nicht unerheblich. 1971 - dem letzten Jahr, für das Gesamtzahlen vorliegen - machten die direkten Steuern 27,3 Prozent aller laufenden und 29,4 Prozent aller Steuereinnahmen aus.

18 Ihr Anteil an den Gesamteinnahmen aller öffentlichen Hände betrug 1971 72,7 Prozent.

19 United Nations Economic Commission for Latin America: Economic Survey of Latin America 1970, New York 1972, S. 50, Tab. 16. Diese Statistik bezieht sich auf den Zeitraum 1966 bis 1969. Extreme stellen Venezuela (67,6 Prozent) und Guatemala (22,5 Prozent) dar. 1967/68 betrug die Relation in Costa Rica 36,3 Prozent.

20 Angeregt durch Carl Föhl, Kritik der progressiven Einkommensbesteuerung, in: Finanzarchiv, NF, Tübingen 1954. Weitere Autoren der sich anschließenden Debatte zu nennen, verbietet der Raum.

21 Damit ist es gleichfalls nicht möglich, wirtschaftspolitische Instrumente zur Beeinflussung der Bedingungen, welche die Ưberwälzung auch der direkten Steuern ermöglichen, zu entwickeln.

22 Die Ley de la Administración Financiera (Nr. 1279 v. 2. 5. 51, of t novelliert) statuiert den erwähnten Grundsatz ausdrücklich in ihrem Artikel 51; auch in Art. 176 der Verfassung sowie in der Ley sobre el Presupuesto Nacional sind entsprechende Bestimmungen enthalten. 
die 6,0 Prozent des Staatshaushaltes erhält. Es erscheint zumindest fraglich, ob der ursprünglich angestrebte Zweck - die Verminderung der jährlichen Verteilungskämpfe und die Stärkung der verfassungsmäßigen Unabhängigkeit der Justiz durch dieses dem Prinzip der Budgetrationalität widersprechende Verfahren erreicht wird. Vor allem aber führen die verschiedenen Formen der Zweckbindung dazu, daß die finanzielle Manövrierfähigkeit des Staates beeinträchtigt wird.

4. Die Steuerlast ist im Vergleich zu anderen Ländern Lateinamerikas von durchschnittlicher Höhe. Bezogen auf das Bruttoinlandsprodukt (Gross Domestic Product) betrugen 1969 sämtliche laufenden Einnahmen 12,7, alle Steuereinnahmen 9,9 Prozent ${ }^{23}$.

Die entsprechenden Werte für $1971^{24}$ sind 14,8 und 12,2 Prozent; sie haben also erheblich zugenommen. Im Vergleich $\mathrm{zu}$ anderen lateinamerikanischen Staaten befindet sich Costa Rica auch hier in einer mittleren Position ${ }^{25}$.

5. Einfluß der Steuerzahler auf die Steuerpolitik.

Das freiheitliche politische System Costa Ricas fördert eine lebendige Diskussion von Steuerfragen. Das zeigt sich etwa an der 1972 erhobenen, von erheblicher Publizität begleiteten Verfassungsklage der Handelskammer gegen das Verbrauchssteuergesetz samt Zusatzverordnungen. Eine Organisation, die dem deutschen Bund der Steuerzahler entspräche und allgemeine bzw. gemeinsame Belange der Steuerzahler dem Staat gegenüber wahrnähme, existiert allerdings nicht; dies hängt auch mit dem geringen Maß an direkten, vom Steuerzahler unmittelbar registrierten Steuern zusammen.

\section{Die Organisation der Steuerverwaltung}

Die gesamte Steuerverwaltung untersteht der dem Finanzministerium eingegliederten Dirección General de Hacienda ${ }^{26}$. Ihr sind zwei zentrale Behörden untergeordnet: die mit der Erhebung der direkten und indirekten Steuern betreute Dirección General de la Tributación Directa und die als oberste Zollbehörde eingerichtete Dirección General de Aduanas. Außerdem ist ihr mit der Oficina de Cobros ein für die Zwangseintreibung von Steuern zuständiges Sonderbüro zugeteilt.

Die Dirección General de la Tributación Directa ${ }^{27}$ verfügt über eine Grundsteuer- und eine für sämtliche anderen Steuerarten zuständige Abteilung. Diese Organisationsstruktur ist wenig funktionsgerecht. Wenn man schon davon abgesehen hat, die Verwaltung der indirekten Steuern einer gesonderten Behörde zu übertragen, so erscheint es doch wenig zweckmäßig, daß die Grundsteuerabteilung für weniger als 5 Prozent, die andere Abteilung jedoch für mehr als 50 Prozent

\footnotetext{
23 Berechnet nach United Nations Statistical Yearbook 1971, S. 560; 1970, S. 649; United Nations Economic Commission for Latin America: Economic Survey of Latin America 1970, New York 1972, S. 160.

24 Eigene Berechnungen.

25 United Nations, Economic Survey of Latin America 1967, New York 1969, S. 107-116. Sieht man von Bundesstaaten $\mathrm{ab}$, deren Einzelstaatenhaushalte das Bild verfälschen, so sind die Extreme im Jahre 1966 Guatemala mit 8,0 bzw. 7,5 Prozent (1966) und Chile mit 22,4 bzw. 20,7 Prozent (1966).

26 Errichtet durch die Ley de Creación de la Dirección General de Hacienda, No. 3022, vom 27. August 1962.

27 Organisation und Zuständigkeiten der Dirección General de la Tributación Directa sind in verschiedenen Erlassen (Decretos Ejecutivos) sowie in einigen materiellen Steuergesetzen, so z. B. im Einkommensteuergesetz, geregelt. Zeitweilig bestand zusätzlich eine für die Administrātion der Verbrauchssteuern zuständige Dirección General de Consumo, die jedoch vor einigen Jahren abgeschafft wurde, (Informe de la Primera Reunión de Directores de Tributación de Centroamérica y Panamá, Bd. II, San José 1967, S. 3 und 6; Maria Teresa Mora Jiménez et al.: Analisis de las Finanzas Públicas y del Sistema Tributario de Costa Rica (hektograf.), San José 1968, S. 9).
} 
der gesamten Steuereinnahmen zuständig ist ${ }^{28}$. Darüber hinaus überrascht das Ungleichgewicht der Zahl der den beiden Verwaltungseinheiten zugewiesenen Bediensteten: So gehören der Grundsteuerabteilung immerhin 119, der anderen Abteilung hingegen nur 200 Beamte an ${ }^{29}$ - eine Relation, die angesichts der unterschiedlichen fiskalischen Bedeutung der von ihnen verwalteten Steuern unausgewogen ist.

Die Generaldirektion ist mit ihren Ämtern in der Hauptstadt angesiedelt. Darüber hinaus sind in jüngster Zeit zwei Regionalbüros in Puntarenas und in Pérez Zeledón errichtet worden, die den Kontakt mit den Steuerzahlern, insbesondere den Unternehmen, verstärken und sonstige Hilfsfunktionen wahrnehmen sollen ${ }^{30}$. Weitere Stellen dieser Art in anderen Landesteilen sind geplant. Die Beschränkung der den Regionalbüros zugewiesenen Aufgaben ist sinnvoll. Eine Dekonzentration der Steuerverwaltung auf die Provinzen mit weitreichender Aufgabendelegation an örtliche Finanzämter wäre mit Rücksicht auf die begrenzten finanziellen Mittel und die geringe Steuerkraft des Hinterlandes verfehlt.

Der Dirección General de Aduanas ${ }^{31}$ unterstehen acht lokale Zollämter, die mit der Erhebung der Zölle und der Überwachung des Warengrenzverkehrs betraut sind.

Verwaltungsakte der Steuerbehörden können bei einem Sondergericht, dem Tribunal Fiscal Administrativo ${ }^{32}$, angefochten werden. Rechtsmittel gegen zollrechtliche Klassifikationsentscheidungen sind dagegen beim Comité Arancelario einzulegen, das aufgrund des Zollabkommens des Gemeinsamen Marktes errichtet worden und mit gerichtsähnlichen Funktionen ausgestattet ist.

\section{Das Verwaltungsverfahren}

Das Verwaltungsverfahren, das in einigen wichtigen Aspekten einheitlich geregelt ist $^{33}$, weist für die Einzelsteuern folgende Merkmale auf:

\section{Einkommensteuer}

Die Steuerzahler sind verpflichtet, sich selbst zu veranlagen. Sie müssen grundsätzlich - auch dann, wenn die Steuer im Lohnsteuereinzugsverfahren einbehalten wird - bei der Dirección General de la Tributación Directa in der Zeit vom 1. Oktober bis zum 30. November (für das vorangegangene Steuerjahr vom 1. Oktober bis 30. September) eine beeidete Erklärung einreichen, in der sie die Steuer unter Berücksichtigung von Freibeträgen und Absetzungen errechnet haben. Von der Pflicht zur Vorlage einer Erlslärung waren bis 1971 natürliche Personen mit

28 Zahlen für 1971: Contraloría General de la Républica, Tipo Programas, a. a. O.

29 Zahlen nach dem Budget für 1973.

30 Dirección General de la Tributación Directa, Memoria Anual 1972, S. 20; als Quellen sind ferner verschiedene persönliche Mitteilungen von Mitarbeitern der Steuerverwaltung zu nennen.

31 Der Aufbau dieser Behörde und der ihr nachgeordneten Zollämter lehnt sich weitgehend an die Regelungen im Código Aduanero Uniforme Centroamericano (CAUCA) und im Reglamento del Código Aduanero Uniforme Centroamericano (RECAUCA) an; er entspricht deshalb in vielen Aspekten der Organisation der Zollverwaltung in Guatemala.

32 Codigo de Normas y Procedimientos Tributarios (Código Tributario), Ley No. 4755, Art. $149-156$.

$33 \mathrm{Im}$ Código Tributario. Die übrigen Verfahrensbestimmungen finden sich in den verschiedenen materiellen Steuergesetzen. 
einem Brutto-Jahreseinkommen unter C 5000 befreit; seitdem ist die Grenze auf C 15000 angehoben worden. Infolgedessen verringerte sich die Zahl der eingereichten Erklärungen von 104917 im Jahre 1971 auf 49850 im Jahre 1972; die Relation zwischen der Anzahl der Erklärungen und den tatsächlichen Steuerleistungen 1970 immerhin schon 75 Prozent - dürfte damit noch günstiger geworden sein ${ }^{34}$.

Die Angaben der Steuerzahler werden in nur wenigen Fällen einer Kontrolle unterzogen. Lediglich bei den größeren Unternehmen mit einem Jahresumsatz von mehr als C 10,0 Mio. finden Betriebsprüfungen statt; bei den übrigen Unternehmen und bei Gehaltsempfängern mit Jahreseinkünften von über $C 70000$ beschränkt sich die Behörde auf eine Kontrolle anhand der ihr verfügbaren schriftlichen Unterlagen. Prüfungen der übrigen Steuerzahler werden wegen des Personalmangels nur ausnahmsweise vorgenommen. Ein systematisches Auswahlverfahren wird bislang nicht praktiziert. Die Steuerzahler können deshalb im allgemeinen damit rechnen, daß ihre Angaben nicht kontrolliert werden. Sofern jedoch die Behörde in den wenigen von ihr untersuchten Fällen zu Beanstandungen Anlaß findet, kann sie die Steuer selbst festsetzen.

Bei den meisten Steuerpflichtigen wird die Einkommensteuer im Wege des Lohnsteuereinzugsverfahrens einbehalien. Gewerbetreibende und Freiberufler müssen vierteljährliche Vorauszahlungen leisten. 1972 beliefen sich die Zahlungsrückstände in 5688 Fällen auf insgesamt mehr als C 31 Mio. In 1698 Fällen schritt die Oficina de Cobros zur Zwangsbeitreibung und erzielte dadurch ein zusätzliches Aufkommen von etwa C 5 Mio. $^{35}$. In diesen Zahlen kommt die laxe Steuermoral zum Ausdruck, der die Verwaltung nicht energisch genug entgegentritt.

Entscheidungen der Steuerbehörde werden nur ausnahmsweise angefochten. So wurden 1972 nur 49 Beschwerden beim Finanzgericht eingelegt ${ }^{36}$.

\section{Umsatzsteuer und Verbrauchssteuer}

Auch die Umsatzsteuer wird aufgrund der Selbstveranlagung erhoben. Gewerbeteibende und Freiberufler, deren Jahresumsatz den Betrag von C 300000 übersteigt, müssen monatlich in einer Erklärung sämtliche Umsätze anmelden, die sie gegenüber ihrerseits nicht steuerpflichtigen Personen getätigt haben. Nur diese Umsätze unterliegen der Abgabepflicht; dadurch wird der Einphasencharakter der Steuer gewahrt.

Kontrollen, insbesondere in Form von Betriebsprüfungen, sind selten und folgen keinem bestimmten System. 1972 wurden 172 von 1538 Fällen untersucht; dabei erzielte die Behörde aufgrund von Steuerneufestsetzungen ein Mehraufkommen von immerhin ca. C 2,7 Mio. ${ }^{37}$.

Der Steuerpflichtige muß bei Vorlage der Erklärung einen Zahlungsnachweis erbringen. Bei Säumnis von mehr als 60 Tagen kann die Verwaltung sein Unternehmen schließen. Dieses Sanktionsmittel hat sich als äußerst wirksam erwiesen: Die

\footnotetext{
34 Dirección General de la Tributación Directa, Estadística Demográfica Fiscal del Impuesto sobre la Renta - Periodo 39, San José 1972, S. 23, sowie mündliche Ergänzungen durch die Oficina de Planeamiento in der Tributación Directa; Dirección General de la Tributación Directa, Memoria Anual 1972, San José 1973, S. 26.

35 Dirección General de la Tributación Directa, Memoria Anual, S. 34

36 Dirección General de la Tributación Directa, Memoria Anual, S. 35.

37 Dirección General de la Tributación Directa, Memoria Anual, S. 13.
} 
Zahlungsrückstände beliefen sich Ende Oktober 1972 auf eine Gesamtsumme von C 4,1 Mio. ${ }^{38}$.

Das Verwaltungsverfahren bei der Verbrauchssteuer, der neben den Importeuren die nicht handwerklich produzierenden Fabrikanten unterliegen, ist ähnlich gestaltet. Die Kontrollen durch die Behörde sind unzulänglich ${ }^{39}$.

\section{Importzölle}

Die Importeure können die Waren entweder bei den Grenzzollämtern oder beim Hauptzollamt in San José deklarieren. In beiden Fällen sind die Lagergebühren äußerst niedrig. Die Firmen schieben deshalb die Zollabfertigung so weit wie möglich hinaus; sie können auf diese Weise ihre eigenen Lager entlasten und sich überdies mit der Entrichtung der Abgabe Zeit lassen.

Im allgemeinen gelingt es den Importeuren, sich mit den Zollbeamten zu arrangieren: 1972 sind bei insgesamt mehr als 162516 Fällen nur 1519 Einsprüche eingelegt worden ${ }^{40}$.

\section{Grundsteuer}

Die Grundsteuer wird nach den Wertangaben der Steuerrolle bemessen ${ }^{41}$. Eine zuverlässige Basis für diese Bewertung fehlt in den meisten Fällen. Der 1964 begonnene Versuch, mit Hilfe eines speziellen Steuerkatasters klare Verhältnisse zu schaffen, scheiterte schon nach wenigen Jahren ${ }^{42}$. Von dem im Aufbau befindlichen, nur mühsam voranschreitenden Allzweckkataster wird die Steuerverwaltung in absehbarer Zeit kaum profitieren können. Die amtlichen Grundstücksschätzer stoßen bei ihren Ermittlungen auf oft unüberwindliche Schwierigkeiten. Unter diesen Umständen muß sich die Behörde im allgemeinen mit den 1962/63 abgegebenen, schon damals viel zu niedrigen und inzwischen längst überholten Selbsteinschätzungen der Grundeigentümer ${ }^{43}$ abfinden. Neubewertungen von Grundstücken erfolgen nur bei Verfügungsgeschäften und bei Änderungen der tatsächlichen Verhältnisse durch äußere Einwirkungen. Der Verwaltung ist es bislang, trotz der gesetzlich vorgesehenen Sanktionsmittel, nicht gelungen, den deutlich spürbaren Steuerwiderstand zu brechen. Viele Grundeigentümer setzen sich gegen ihre Zahlungsverpflichtungen mit Erfolg zur Wehr. Ende 1972 beliefen sich die Außenstände auf insgesamt mehr als C 48 Mio. $^{44}$ - einen Betrag, der sogar das jährliche Aufkommen übersteigt.

\footnotetext{
38 Dirección General de la Tributación Directa, Memoria Anual, S. 17.

39 Dirección General de la Tributación Directa, Memoria Anual, S. 12 f.

40 Mündliche Auskunft in der Dirección General de Aduanas.

41 S. dazu im einzelnen Organización de Estados Americanos/Banco Interamericano de Desarrollo, Sistemas Tributarios de América Latina: Costa Rica, Washington 1965, S. 27-30.

42 Dirección General de la Tributación Directa: Sobre la problemático del Impuesto Territorial y la Responsabilidad de la Administración Tributaria (hektograf.), San José 1972, S. 4 f.

43 Aufgrund Gesetz Nr. 3026.

44 Dirección General de la Tributación Directa: Memoria, a. a. O., S. 17.
} 


\section{B. GUATEMALA}

\section{Einführung}

Mit einer Bevölkerungszahi von etwa sechs Millionen und einer im Vergleich zu den meisten Nachbarländern weitentwickelten verarbeitenden Industrie nimmt Guatemala in der zentralamerikanischen Wirtschaftsgemeinschaft eine führende Stellung ein, die an die lange Geschichte seiner Vorherrschaft innerhalb der mittelamerikanischen Ländergruppe anknüpft.

Trotz der staatlichen Entwicklungs- und Diversifikationsbestrebungen hängt die Wirtschaft noch immer von der Ausfuhr und damit von den Weltmarktpreisen seiner vier Haupt-Agrarprodukte (Kaffee, Baumwolle, Zucker und Bananen) ab. Allein der Kaffee macht $34 \%$ des Gesamtexportwertes aus. Auf die weiterhin dominierende Plantagenwirtschaft ist es unter anderem zurückzuführen, daß die bestehende Agrarbesitzstruktur nicht angetastet wird. Zusammen mit der ethnischen Klüftung des Landes stellen diese ungleichen Grundbesitzverhältnisse das schwerste Problem Guatemalas dar. Nach dem erfolglosen Versuch einer durchgreifenden Agrarreform 1954 stützt sich die Landwirtschaftspolitik seit 1956 auf ein Gesetz, das die Probleme der landlosen Bauern und der Minifundien ausschließlich durch Urbarmachung und Verteilung nichtbewirtschafteten Bodens lösen soll. Man hat errechnet, daß es 230 Jahre dauern würde, bis die gesamte Landbevölkerung auf diese Weise mit Ackerland versorgt wäre. Auch die Aufgabe des Plantagenbesitzes durch die United Fruit Company Anfang 1973 hat nichts an der hohen Konzentration des landwirtschaftlich nutzbaren Bodens in der Hand weniger Großgrundbesitzer geändert.

Eng verbunden mit der Landbesitzproblematik ist die Spaltung des Landes in seine beiden größten Bevölkerungsgruppen: die Indios (ca. $53 \%$ der Bevölkerung) im Nordwesten und die "Ladinos“ (ca. 30\%) in den mestizischen Provinzen des Südens und Südostens. Seit der Unabhängigkeit vor 150 Jahren sind Erfolge bei der wirtschaftlichen, kulturellen und politischen Eingliederung der indianischen Bevölkerungsmajorität in die guatemaltekische Gesellschaft kaum erzielt worden. Wegen der starken sozialen Trennung zwischen Stadt und Land bleibt die politische Partizipation auf eine kleine Bevölkerungsgruppe - vor allem die Mittelund Oberschichten der fünf Städte mit mehr als 25000 Einwohnern - beschränkt.

Die seit 1966 zunehmende Guerilla-Tätigkeit im südöstlichen Hinterland und in der Hauptstadt erreichte vor den Wahlen 1970 ihren Höhepunkt mit der Ermordung des deutschen Botschafters von Spreti. Diese Unruhen trugen zu dem Wahlsieg der konservativen Gruppierungen Partido Institucional Democrático (PID) und Movimiento de Liberación Nacional (MLN) bei. Auch in der gegenwärtigen, von denselben politischen Gruppierungen getragenen Regierung sind - wenngleich indirekt - wieder die Streitkräfte an der Exekutive beteiligt, die mit Unterbrechungen seit mehr als 100 Jahren in die Staatsführung eingegriffen haben. Allerdings ist die Machtstellung der Armee inzwischen von den "finqueros" unabhängiger geworden, da sich das Offizierscorps durch die Zuweisung von Ländereien wirtschaftlich emanzipieren konnte und mit dem Kampf gegen die Untergrundbewegung eine neue anerkannte Rolle gefunden hat.

Guatemala ist Republik mit einem Präsidialsystem und einer zentral staatlichen Verwaltungsstruktur. Seine Verwaltung ist ganz auf die Hauptstadt zugeschnitten 
und läßt den 22 Bezirken und den Munizipien nur beschränkte Befugnisse. Eines der Hauptprobleme ist der Mangel einer qualifizierten und unbestechlichen, nach Eignungskriterien rekrutierten Beamtenschaft. Angesichts nur gering ausgebildeter demokratischer Traditionen und der tiefen sozialen und ethnischen Gegensätze dürfte ein System repräsentativer Demokratie in absehbarer Zeit - wenn überhaupt -- nur sehr beschränkt funktionsfähig sein.

\section{Das Steuersystem}

Stärker noch als in Costa Rica ist in Guatemala eine Zersplitterung des materiellen Steuerrechts zu beobachten. Angesichts der unübersehbaren Vielfalt der Abgaben werden nur die fiskalisch wichtigen (Einkommen- und Umsatzsteuer, Verbrauchssteuer auf Erdölderivate, Importzölle) sowie die Grundsteuer behandelt. Damit sind fast drei Viertel des gesamten Steueraufkommens erfaßt ${ }^{45}$.

Der Einkommensteuer ${ }^{46}$ unterliegen gleichermaßen natürliche und juristische Personen. Bananen-, Bergwèrks- und Ölgesellschaften sind von der Besteuerung freigestellt, werden jedoch einer besonderen, vertraglich geregelten Abgabepflicht unterworfen. Eine Differenzierung des Tarifs nach der Rechtsnatur der Steuerpflichtigen, wie sie in den meisten anderen Ländern üblich ist, gibt es nicht. Dividendenausschüttungen werden nur bei der Kapitalgesellschaft, nicht aber zusätzlich beim Anteilseigner versteuert.

Die Steuerpflicht beginnt erst bei einem Jahreseinkommen von $Q^{47} 1800$; dies ist für guatemaltekische Verhältnisse ein beachtlich hoher Freibetrag. Der Großgrundbesitz wird in krasser Weise bevorzugt. Bruttoeinnahmen, die ausschließlich aus landwirtschaftlicher Tätigkeit bezogen werden, bleiben steuerfrei, sofern sie die Grenze von jährlich Q 15000 nicht überschreiten. Diese Regelung läßt die starke Stellung der Großgrundbesitzer im politischen System Guatemalas erkennen. Die Steuersätze sind ungewöhnlich niedrig und weisen eine geringe Progression auf. So müssen etwa bei einem zu versteuernden Jahreseinkommen von Q 4500 6,75 Prozent, bei einem Einkommen von Q 21000 nur 15 Prozent an Steuern entrichtet werden; die Progressionszone endet bei einem Jahreseinkommen von Q $500000 \mathrm{mit}$ 48 Prozent. Immerhin sind die Erträge aus der Einkommensteuer im letzten Jahr stark angestiegen: von Q 6,6 Mio. im Jahre 1960 auf Q 20,5 Mio. im Jahre 1971. Trotzdem ist der Anteil der Einkommensteuer am Gesamtsteueraufkommen in den vergangenen Jahren mit ca. 14 Prozent gleich geblieben ${ }^{48}$.

Die Umsatzsteuer ${ }^{49}$ erfaßt als Brutto-Allphasen-Steuer in erster Linie den Umsatz von Gütern und Dienstleistungen; dabei wird ein allgemeiner Steuersatz von 1,5

\footnotetext{
45 Dirección Técnica del Presupuesto, Presupuesto de ingresos y egresos del estado Ejercicio fiscal 1973.

46 Gesetz Nr. 229 vom 28. 11. 1964 mit Durchführungsverordnungen. J. Lucas Caballeros: Estudio sobre el impuesto sobre la renta en Guatemala, Univ. San Carlos de Guatemala, hektografiert, Guatemala 1967; Secretaría General del Consejo Nacional de Planificación Económica: Plan de Desarrollo 1971-75 (im folgenden "Entwicklungsplan 1971-75“): Anexo I, Tomo II: Aspectos administrativos, reformas y proyecciones (Programa tributario del gobierno central, 1971-75, Abschnitt III: El Impuesto reformas y proyecciones (Programa tributario del gobierno central, 1971-75, Abschnitt III: El Impuesto
sobre la Renta); Secretaria Permanente para la Integración Económica de Centroamérica: El Desarrollo integrado de Controamérica en la presente década: Bases y propuestas para el perfeccionamiento y la reestructuración del Mercado Común Centroaméricano, Estudio No. 10: Las finanzas públicas y la integración (Programa de Armonización Tributaría) (Dokument SIECA/72-VII-6-36), Guatemala 1972.

$471 \mathrm{Q}$ (Quetzal = 1 US-\$ (1973).

48 Berechnungen nach Dirección de Contabilidad del Estado; Cuadros de la situación fiscal y financiera del Estado correspondiente al mes de diciembre de 1972 (bzw. febrero de 1973), Guatemala, Maschinenschrift, und dem Haushaltsplan 1973, Presupuesto 1973, a. a. O.

49 Decreto Legislativo 1831 von 1932.
} 
Prozent zugrundegelegt. Die Einführung einer Mehrwertsteuer, die schon mit Rücksicht auf den Zentralamerikanischen Gemeinsamen Markt geboten wäre, scheiterte 1967 am Widerstand einflußreicher Kreise aus Handel und Industrie. Die Umsatzsteuer ist inzwischen die fiskalisch bedeutendste Einnahmeart. Gegenüber einem Aufkommen von nur Q 5,5 Mio. im Jahre 1960 fielen 1972 Erträge von Q 40,5 Mio. an ${ }^{50}$. Damit hat sich der Anteil an den gesamten Steuereinnahmen von 7,1 Prozent (1960) auf 26,1 Prozent (1972) erhöht.

Die Bedeutung der Importzölle geht seit Errichtung des Zentralamerikanischen Gemeinsamen Markts relativ zurück. Der Markt verfügt über einen gemeinsamen Außenzoll. Die meisten Einfuhrgüter aus Mitgliedsländern sind von Zollbelastungen freigestellt ${ }^{51}$. Das hat zu einer erheblichen Ausweitung des Handels innerhalb der Wirtschaftsgemeinschaft geführt. Infolgedessen ist der Anteil der Importzölle am gesamten Steueraufkommen von 39,8 Prozent im Jahre 1960 auf 23,9 Prozent im Jahre 1972 gesunken ${ }^{52}$.

Die Verbrauchssteuer auf Erdölderivate ${ }^{53}$ erfaßt in erster Linie den inländischen Verbrauch von Raffineriererzeugnissen. Zahlreiche, unter entwicklungs- und sozialpolitischen Gesichtspunkten nicht gerechtfertigte Befreiungen (in verschiedenen Spezialvorschriften) erschweren die Wirksamkeit dieser Steuer. Immerhin ist das Aufkommen nicht unerheblich gestiegen: von Q 6,8 Mio. im Jahre 1967 auf Q 13,3 Mio. im Jahre 1972. Damit hat sich der Anteil an den gesamten Steuereinnahmen von 7,4 Prozent (1967) auf 8,5 Prozent (1972) erhöht ${ }^{54}$.

Die Besteuerung von Grund und Boden hat zu keiner Zeit spürbare fiskalische und soziale Wirkungen gehabt. Der Grundsteuer ${ }^{55}$ unterliegen Grundstücke im Wert von mehr als Q 100. Bei einem Wert bis zu Q 20000 beträgt der Steuersatz nur 3 Promille, ansonsten 6 Promille jährlich. Bei einem derart niedrigen Tarif bleiben erhebliche Steuerreserven unausgeschöpft. Darin zeigt sich erneut die Privilegierung des Großgrundbesitzes. 1971 erbrachte die Steuer nur Q 4,84 Mio. (4,3 Prozent des Gesamtaufkommens), 1972 6,2 Mio. (5,0 Prozent) ${ }^{56}$.

Mit der Einführung der Steuer auf ungenutztes Land ${ }^{57}$ sollte vor allem die Landreform gefördert werden. Die Erträge aus dieser Steuer sind unbedeutend und zeigen, wie wenig die gegenwärtig maßgebenden politischen Gruppen an der Verwirklichung der der gesetzlichen Regelung zugrundeliegenden sozialpolitischen Zielsetzungen interessiert sind. 1968 wurden Q 8819, 1970 Q 56806 aufgebracht $^{58}$. In den meisten Verwaltungsbezirken gelangt die Steuer überhaupt nicht zur Anwendung. Die Erträge sind zweckgebunden; sie fließen dem wenig bedeutenden Instituto Nacional de Transformación Agraría zu.

Die Analyse des Steuersystems insgesamt ergibt:

1. Die indirekten Steuern überwiegen bei weitem. Sie machten im Jahre 1972 80,83 Prozent der Steuereinnahmen und 67,8 Prozent der laufenden Einnahmen

50 Dirección de Contabilidad: Situación fiscal, Diciembre de 1972, a. a. O., weniger als im Haushalt veranschlagt.

51 Alan Cohen, El desarrollo económico en Guatemala, Guatemala 1970, S. 222.

52 Eigene Berechnungen auf der Basis von Zahlen in Cohen, op. cit., Tabla A 5 J. I., Lopez Coarchita, El sistema tributario de Guatemala y su posible restructuración (Üniv. San Carlos de Guatemala, Tesis de grado), Guatemala 1971, Anexo 1; Haushaltsplan 1973; Monatsausweis der Contabilidad, Dezember 1972; Bruttosozialprodukt 1972 nach Angaben der Zentralbank durch die Verfasser geschätzt.

53 Decreto-Ley 58 von 1963.

54 s. Anmerkung 52.

55 Decreto Legislativo 1153 von 1921.

56 Dirección de Contabilidad, Situación fiscal, Diciembre de 1972, Febrero de 1973.

57 Ley de Transformación Agraría.

58 C. H. Orantes Martinez: El impuesto territorial en Guatemala (Univ. Rafael Landivar, Tesis de grado), Guatemala 1971, Anexo 5, Anexo 8. 
aus $^{59}$; die Ansätze im Haushalt 1973 belaufen sich auf 80,80 bzw. 68,93 Prozent. Entsprechend gering ist der Anteil der direkten Besteuerung. Nach einer Aufstellung der Vereinten Nationen für die Jahre 1966-196960 hat Guatemala von 18 untersuchten lateinamerikanischen Ländern den geringsten Anteil direkter Steuern. Damit ist - folgt man der klassischen Theorie - das Steuersystem stark regressiv. Es ist deshalb ungerecht.

2. Das staatliche Einnahmesystem ist im Verhältnis zum Sozialprodukt unelastisch $^{61}$. Bei gleichen progressiven Tarifen der Einzelsteuern müßten normalerweise mit wachsender Wirtschaft die Erträge überproportional zunehmen. In Guatemala ist das nicht der Fall; die Anteile der staatlichen Einnahmen am Bruttosozialprodukt sind von 1952 bis 1965 praktisch gleich geblieben ${ }^{62}$ (10,2 gegen 10,6 Prozent). Der Entwicklungsplan 1971-75 berechnet für die Jahre 1961-68, unter der Annahme einer neutralen Steuerverwaltung, eine Elastizität von 0,33 im Verhältnis zum Bruttoinlandsprodukt; dieser Wert liegt weit unter der Schwelle von 1,0, der für Proportionalität des Wachstums beider Größen stünde. Die Konstanz des Staatsanteils ist also nur durch mehrfache Anhebungen der Steuertarife erreicht worden ${ }^{63}$.

3. Die Steuerlastquote ist niedrig: bezogen auf das Bruttosozialprodukt betrug sie 1972 ca. 6,45 Prozent. Der Anteil der gesamten laufenden Einnahmen lag bei 7,68 Prozent; unter Einbeziehung auch der Kapitaleinkünfte errechnet sich ein Staatsanteil von 10,77 Prozent; dieser niedrige Wert hat die Staatsfinanzen bei der großen Elastizität der Ausgaben in eine angespannte Lage gebracht. Die Wirtschaftsübersicht der Vereinten Nationen für Lateinamerika für $1967^{64}$ enthält Angaben über den Staatsanteil einiger lateinamerikanischer Länder: Guatemala hat danach von fünf untersuchten Einheitsstaaten den geringsten Anteil ${ }^{65}$.

4. Es gibt zahllose Steuervorschriften. Die meisten betreffen Bagatellsteuern oder Abgaben, die keinen Ertrag bringen. Die Vorschriften sind vielfach nicht aufeinander abgestimmt; gelegentlich wird eine vom Gesetz bereits erfaßte Materie durch neue Normen, ohne Bezug auf frühere Bestimmungen, „geregelt“. Man kann daher nur bedingt von einem Steuersystem sprechen ${ }^{66}$.

5. Das Steuersystem ist durch umfangreiche und sachlich nicht gerechtfertigte Durchbrechungen und Ausnahmeregelungen mit einem weiten Ermessensspielraum der Exekutive gekennzeichnet.

6. Die Komplexität und Unübersichtlichkeit des Systems macht seine konsequente Anwendung für die Verwaltung unmöglich. Die Behörden selbst sind nicht in der Lage, die geltenden Steuern zu überblicken ${ }^{67}$.

59 Dirección de Contabilidad: Situación fiscal, Diciembre de 1972, a. a. O.

60 U. N. Economic Commission for Latin America (ECLA)/Comisión Económica para América Latina (CEPAL): Economic Survey of Latin America 1970, New York 1972, Tab. XVI, S. 50.

61 Lopez Coarchita: Sistema, a. a. O., S. 134; im Detail s. Entwicklungsplan 1971-75, Annex I, Band II, S. $1-3 \mathrm{ff}$.

62 Cohen: Desarrollo, a. a. O., Tabla A-5.

63 Beispiele im Entwicklungsplan 1971-75, S. III-18 für die Einkommensteuer, Elastizität mit Tarifänderungen 1,29 (Band II, 157), was aber für 1972 nicht gilt. So wurde dem Parlament von der Regierung auch im August 1974 ein Steueränderungsgesetz vorgelegt, das teilweise beträchtliche Tariferhöhungen u. a. auch im August 1974 ein Steueränderungsgesetz vorge

64 U. N. Economic Commission for Latin America (ECLA)/Comisión Económico para América Latina (CEPAL): Economic Survey of Latin America 1967, New York 1969, S. 107-116.

$65 \mathrm{Im}$ Falle Mexikos ergibt sich zwar ein noch niedrigerer Wert, dies ist jedoch darauf zurückzuführen, daß die Haushalte der Einzelstaaten nicht berücksichtigt wurden.

66 So erzielt die wichtigste Steuerbehörde, die Dirección General de Rentas Internas, nur Erträge aus 34 von vermutlich weit über 100 Steuern.

67 Aufschlußreich ist das oben kurz gestreifte Ley del Impuesto sobre las Tierras Ociosas, das ganz offen als unanwendbar bezeichnet wird. 
Für den Steuerzahler ergibt sich hieraus eine ambivalente Situation: verfügt er über persönliches Geschick, politische Protektion, Kenntnis der Gesetze und sonstige Einwirkungsmöglichkeiten (Bestechung), so kann er die vielen Lücken und Widersprüche im Steuerrecht und die Mängel der Verwaltung zu seinem Vorteil nutzen. Ansonsten ist er dem Wohlwollen oder der Willkür der Verwaltung ausgesetzt.

7. Die Einzelsteuern weisen zahlreiche Nachteile auf, die sich auf das System insgesamt auswirken und vor allem die wirtschaftliche Neutralität und die steuerliche Gerechtigkeit beeinträchtigen. Zu den schwerwiegenden Fehlern zählt die Tatsache, daß die Umsatzsteuer als kumulative Allphasensteuer die Kleinunternehmen benachteiligt. Bei der Einkommensteuer führt die Besteuerung des Gewinns ausschließlich bei der Kapitalgesellschaft und zum gleichen Tarif wie bei Einkünften natürlicher Personen zu Verzerrungen. Großaktionäre werden dadurch privilegiert, Kleinaktionäre werden nicht nur gegenüber den großen Anteilseignern, sondern auch gegenüber anderen Personen mit sonstigen Einkünften benachteiligt. Die großzügig bemessenen Freibeträge bei der Einkommensteuer nehmen darüber hinaus den größten Teil des Mittelstandes und des mittleren Gutsbesitzes von der direkten Besteuerung weitgehend aus. All diese Tatsachen sind bekannt; seit Jahren werden Reformen vorgeschlagen, die jedoch bisher nicht in die Wirklichkeit umgesetzt worden sind.

8. Das Steuersystem hat weder in direkter Form noch auf dem Umweg über die Erhöhung der Sparneigung Kapitalbildung und Industrieentwicklung geför$\operatorname{dert}^{68}$.

9. Nicht wenige Steuern werden als "rentas consignadas ${ }^{69}$ “ von vornherein bestimmten Zwecken zugeführt. Sie beliefen sich 1972 auf Q 16,4 Mio. und damit auf 8,9 Prozent der laufenden Einnahmen. Es handelt sich z. B. um eine 11prozentige Sondersteuer auf internationale Flugpassagen zur Verbesserung der technischen Anlagen des Flugverkehrs, um eine Zusatzbelastung des Kaffee-Exports zur Strukturverbesserung der Kaffeebaugebiete u. $\ddot{a}$.

10. Die Pressure Groups, insbesondere Verbände des Handels, der Industrie und der Landwirtschaft, üben starken Einfluß auf Steuergesetzgebung und Verwaltungspraxis aus ${ }^{70}$. Eine rege öffentliche Meinung, die mit Anteilnahme und Sachkenntnis Steuer- und Haushaltsfragen diskutierte, besteht in Guatemala nicht. Einflußnahmen vollziehen sich daher im Halbdunkel öffentlich unkontrollierter Räume. Die Macht der verschiedenen Gruppen dürfte so groß sein, daß z. Z. tiefgreifende Steuerreformen nicht ohne ihre stillschweigende Duldung, wenn nicht sogar aktive Kooperation, realisierbar sind.

\section{Die Organisation der Steuerverwaltung}

Der Dirección General de Rentas Internas untersteht die Verwaltung nahezu sämtlicher direkter und indirekter Steuern. Die Dirección General de Aduanas ist für

\footnotetext{
$68 \mathrm{Vgl}$. die Werke über Industrieentwicklung: C. A. Molina Woolford, Protección arancelaria y leyes de fomento industrial en Guatemala: su aplicación y resultados (Universidad San Carlos de Guatemala, tesis de grado), Guatemala 1970; A. Guerra Borges, Evaluación de la política de fomento industrial en Guatemala. Guatemala 1971; A. Pimentel Rodriguez, Los incentivos fiscales y el desarrollo industrial de Guatemala (Universidad San Carlos de Guatemala, tesis de grado), Guatemala 1969; A. M. Ovelle de Uribe, Política arancelaria y desarrollo económico; Introducción al análisis de la política arancelaria en los paises subdesarrollados, con especial referencia al caso de Guatemala en el período $1959-69$. Guatemala 1970.

69 In den Kassenausweisen - Dirección de Contabilidad: Situación fiscal, Diciembre de 1972, Febrero de 1973 , a. a. O.
} 
die Erhebung der Zölle zuständig. Beide Behörden sind dem Finanzministerium eingegliedert ${ }^{71}$.

Die Dirección General de Rentas Internas ist 1972 durch Zusammenlegung zweier getrennter Oberbehörden gebildet worden. Diese Reformmaßnahme ermöglichte eine Straffung der Organisation und der Arbeitsweise. Doch haben sich bis heute noch nicht sämtliche Verwaltungseinheiten auf ihre veränderten Aufgaben eingestellt. Die streng hierarchisch aufgebaute Behörde ist in Departamentos geglie$\operatorname{dert}^{72}$. Diese Departamentos umfassen mehrere Secciones als Unterabteilungen, die ihrerseits aus Divisiones - diese gelegentlich in Unidades unterteilt - bestehen. Die für die Steuererhebung zuständigen drei Departamentos sind nicht nach Steuerarten, sondern nach Funktionen (Steuereinziehung, Erfassung der Steuerzahler, Kontrolle der Steuererklärungen) gegliedert; erst auf der Ebene der untersten Verwaltungseinheiten, der Divisiones und Unidades, setzt die Kompetenzzuordnung nach unterschiedlichen Steuern ein. Bei dieser komplizierten Organisationsstruktur treten nicht unerhebliche Schwierigkeiten bei der Abgrenzung von $\mathrm{Zu}-$ ständigkeiten auf.

Über die in der Hauptstadt konzentrierten Ämter der Generaldirektion hinaus bestehen in 21 der insgesamt 22 Verwaltungsbezirke des Landes (ausgenommen ist Guatemala-Stadt) örtliche Finanzämter. Sie sind formal allzuständig, könnten also Steuererklärungen kontrollieren, Betriebsprüfungen vornehmen usw. Dazu sind sie jedoch wegen ihrer unzureichenden personellen und materiellen Ausstattung im allgemeinen nicht imstande; sie leiten deshalb die meisten Fälle zur Bearbeitung an die Zentralbehörde weiter. Davon abgesehen können die Steuerzahler des Hinterlandes die Generaldirektion von sich aus als Behörde ihrer Wahl bestimmen, indem sie ihren steuerlichen Wohnsitz in die Hauptstadt verlegen und auf diese Weise das örtlich an sich zuständige Finanzamt umgehen. Die gegenwärtige Funktionsaufteilung ist infolgedessen wenig geeignet, zu einer wirksamen Dekonzentration und Regionalisierung der Steuerverwaltung beizutragen.

Die Dirección General de Aduanas, die für die Zollverwaltung zuständige oberste Behörde, ist gleichfalls hierarchisch gegliedert und weist drei Departamentos auf ${ }^{73}$. Ihr sind die 18 örtlichen Zollämter unterstellt. Die Erhebung der Zölle sowie die Überprüfung und Überwachung der Ein- und Ausfuhr sind Sache der lokalen Behörden. Zur Erfüllung ihrer Aufgaben werden ihnen Beamte der Generaldirektion zugeteilt, die jeweils dem örtlichen Verwaltungschef unterstehen.

Ein für Steuerfragen zuständiges Sondergericht existiert nicht. Klagen gegen Verwaltungsakte der Steuerbehörden sind beim allgemeinen Verwaltungsgericht einzulegen. Soweit es sich jedoch um Rechtsmittel gegen zollrechtliche Klassifikationsentscheidungen handelt, ist nach dem Zolabkommen des Gemeinsamen Marktes, nicht anders als in Costa Rica, eine gerichtsähnliche Sonderbehörde, das Comité Arancelario, in erster und letzter Instanz zuständig.

70 Erneut sei auf die Umstände des Mißlingens der Einführung der Mehrwertsteuer hingewiesen.

71 Hierzu und zum folgenden siehe Ley del Ministerio de Finanzas Públicas, Decreto No. 106-71 vom 15. 12. 1971; Reglamento de la Ley del Ministerio de Finanzas Públicas vom 16. 2. 1972 sowie die vom Finanzminister erlassenen Reglamentos Internos der verschiedenen Unterbehörden (Dependencias) des Ministeriums. Durch diese Vorschriften ist die Organisation der Finanzverwaltung zum erstenmal ein-
heitlich, wenn auch nicht erschöpfend, geregelt worden.

72 unpublizierter interner Organisationsplan der Dirección (Reglamento Interno).

73 Der Behördenaufbau richtet sich nach dem Reglamento Interno de la Dirección General de Aduanas, das weitgehend auf den Organisationsvorschriften des Código Aduanero Uniforme Centroamericano (CAUCA) und des Reglamento del Código Aduanero Uniforme Centroamericano (RECAUCA) aufbaut. CAUCA und RECAUCA beruhen auf zwischenstaatlichen Vereinbarungen, die in den Mitgliedsländern als nationalstaatliche Regelungen einheitlich gelten. 


\section{Das Verwaltungsverfahren}

Ein der deutschen Reichsabgabenordnung vergleichbares, die Rechte und Pflichten der Steuerzahler und das Besteuerungsverfahren einheitlich regelndes Gesetz fehlt. Maßgeblich bleiben deshalb vorläufig die in den materiellen Steuergesetzen mühsam aufzufindenden und vielfach widersprüchlichen Verfahrensbestimmungen.

\section{Einkommensteuer}

Die Steuerveranlagung ist Sache des Steuerpflichtigen selbst. Er bedient sich dabei einer von ihm zu beeidenden Erklärung. Natürliche Personen, deren jährliches Bruttoeinkommen den Betrag von Q 6000 nicht übersteigt, sind von der Pflicht zur Vorlage einer Erklärung freigestellt; sie werden zumeist im Lohnsteuerabzugsverfahren erfaßt. Es erscheint wenig sinnvoll, daß die Pflicht zur Vorlage einer Steuererklärung - und damit de facto die Steuerzahlung - von der Höhe des erzielten Einkommens statt von der Einkunftsart abhängig gemacht wird ${ }^{74} 1972$ wurden 45443 Erklärungen eingereicht; doch fiel nur in 15750 Fällen (= 34 Prozent) überhaupt eine Steuer $a^{75}$. Wie hoch die Zahl derjenigen ist, die sich der Erklärungspflicht entziehen, war nicht zu ermitteln. Andeutungen über das Ausmaß der Steuerhinterziehungen lassen aber vermuten, daß die Dunkelziffer sehr hoch ausfällt. Der Verwaltung ist es bislang mangels geeigneten Personals und nicht zuletzt unter dem Druck einflußreicher Interessengruppen nicht gelungen, an diesem Zustand etwas zu ändern.

Die Steuererklärungen sind der örtlich zuständigen Behörde zu übermitteln. Die in der Dirección General zu bearbeitenden Erklärungen werden routinemäßig und folglich nur oberflächlich überprüft. Eine systematische Stichprobenauswahl unterbleibt. Querkontrollen, etwa anhand der Umsatzsteuerkarten, finden nur gelegentlich statt. Werden, wie im Regelfall, keine Mängel festgestellt und hat der Steuerpflichtige die von ihm errechnete Steuer gezahlt, so ist der Besteuerungsvorgang abgeschlossen; die Erklärung wird im Archiv abgelegt. Falls jedoch bei der Kontrolle ausnahmsweise Mängel beobachtet werden, geht die Behörde der Sache nach und setzt die Steuer aufgrund eigener Ermittlungen, ggf. im Wege der Schätzung, fest. Unternehmen mit einem Jahresumsatz von mehr als Q 100000 werden einer Betriebsprüfung unterzogen.

Soweit die örtlichen Finanzämter selbst Steuererklärungen bearbeiten, beschränken sie sich, schon wegen der mangelhaften Personalausstattung, zumeist auf eine flüchtige Prüfung.

Der Steuerpflichtige muß bei der Vorlage der Erklärung die von ihm selbst errechnete Steuer zahlen oder den Nachweis über die bereits erfolgte Zahlung erbringen. Eine Pflicht zur Vorauszahlung mit bestimmten Abschlagsquoten, wie es bei Gewerbetreibenden und Freiberuflern angebracht wäre, besteht nicht. Dadurch werden diejenigen, die die Steuer im Wege des Lohnsteuereinzugsverfahrens entrichten, benachteiligt. Kommt der Steuerzahler seinen Verpflichtungen zur Vorlage

\footnotetext{
74 Von den Angehörigen bestimmter Berufsgruppen, wie z. B. den Freiberuflern und den Gewerbetreibenden, sollte ausnahmslos die Vorlage einer Erklärung verlangt werden (so auch der Entwicklungsplan 1971-75, Anexo I, Tomo II, III - 8, f).

75 Mündliche Auskunft des Departamento Control de Contribuyentes; Dirección General de Rentas Internas, Informe Técnico 1972, Anexos e, f und 24.
} 
der Erklärung und zur Zahlung der Steuer nicht nach, so kann die Verwaltung Sanktionen (Zinszuschlag, Geldbuße, Zwangsbeitreibung o. ä.) verhängen. Von diesen Möglichkeiten macht sie jedoch nur sehr zurückhaltend Gebrauch. Das gilt vor

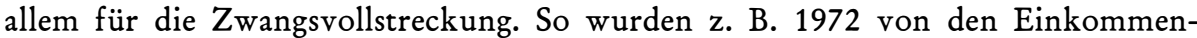
steuererträgen in Höhe von insgesamt Q 23309509 nur Q 1483 zwangsweise beigetrieben ${ }^{76}$. In diesen Tatsachen schlägt sich die geringe Effizienz der Steuerverwaltung nieder. Dabei wirken sich allerdings nicht nur organisatorische und personelle Schwächen aus. In einem politischen Kontext, der den Steuerbehörden die Verwirklichung des gesetzlichen Auftrags strukturell erschwert, stößt die Verwaltung im Bereich der Einkommensteuer sehr bald an die Grenzen ihrer Möglichkeiten.

Gegen Verwaltungsakte der Steuerbehörden kann der Beschwerte Widerspruch einlegen ${ }^{77}$. Gibt die Dirección General dem Widerspruch nicht statt, so steht der Klageweg zum allgemeinen Verwaltungsgericht, mit der Möglichkeit der Berufung beim Obersten Gerichtshof, offen. Da die Steuerbehörden bei der Durchsetzung des staatlichen Steueranspruchs sehr zurückhaltend vorgehen und es vorziehen, sich mit dem Steuerpflichtigen zu arrangieren, ist die Zahl der Rechtsbehelfe äußerst gering. So ist 1972 nur in insgesamt 68 Fällen Widerspruch eingelegt worden ${ }^{78}$.

\section{Umsatzsteuer}

Wer gewohnheitsmäßig Güter oder Dienstleistungen verkauft, ist zur Abgabe einer beeideten Umsatzsteuererklärung verpflichtet. Diese ist jeweils für ein Kalendervierteljahr vorzulegen. Über die Anzahl der bei den Behörden eingehenden Erklärungen sind Informationen nicht erhältlich. Eine gründliche Überprüfung des Wahrheitsgehalts der Erklärung unterbleibt im allgemeinen. Nur gelegentlich werden Inspektionen im Gewerbebetrieb vorgenommen. Die Verwaltung stellt zumeist lediglich fest, ob die in Betracht kommenden Steuerpflichtigen überhaupt eine Erklärung abgegeben haben. Der Steuerzahler kann also auch hier nahezu gewiß sein, daß seine Angaben nicht in Zweifel gezogen werden und es mit der Selbstveranlagung sein Bewenden hat. An sich notwendige Querkontrollen, so z. B. unter Heranziehung der Einkommensteuererklärung, stoßen wegen des Fehlens einheitlicher Steuernummern auf Schwierigkeiten.

Der Steuerpflichtige muß die Steuer bei Vorlage der Erklärung entrichten. Erst dann wird ihm das Original der Erklärung ausgehändigt, das er an sichtbarer Stelle in seinen Geschäftsräumen aufzubewahren hat; dies ist eine der wenigen wirksamen Kontrollmaßnahmen. Gegen die Entscheidung der Behörden kann der Betroffene Widerspruch und ggf. Anfechtungsklage beim allgemeinen Verwaltungsgericht erheben.

Sofern es sich nicht um den gewohnheitsmäßigen Umsatz von Gütern und Dienstleistungen handelt, wird die Steuer durch Verwendung von Stempelpapier oder Steuermarken entrichtet ${ }^{79}$. Auf diese altertümliche, aber doch einfache und

76 Dirección General de Rentas Internas; Informe Técnico 1972, Guatemala 1972, S. 70.

77 Art. 70 Ley de lo Contencioso-Administrativo.

78 Mündliche Auskunft des Deparamento, Control de Contribuyentes.

79 Art. 5 Gesetz 1831. 
effektive Weise, die man häufig in lateinamerikanischen Ländern antrifft, kann die Verwaltung mit verhältnismäßig geringem Aufwand die bei der Ausfertigung amtlicher Dokumente und bei der Vornahme bestimmter juristischer Akte anfallenden Steuern einziehen.

\section{Verbrauchssteuer auf Erdölderivate}

Die Regelung des Verwaltungsverfahrens und der behördlichen Kompetenzen ist widersprüchlich, unklar und unvollständig. Mit der Steuererhebung sind nicht die eigentlichen Steuerbehörden, sondern die Staatskasse und die Dirección General de Minería e Hidrocárburos betraut. Diese Zersplitterung der Zuständigkeiten hat zur Folge, daß eine wirksame Kontrolle unterbleibt.

\section{Importzölle}

Die Importeure können die eingeführten Waren wahlweise in den Grenzzollämtern oder in den Zollämtern Guatemala-Stadt und Quezaltenango deklarieren. Für die Lagerung in den Grenzzollämtern werden keine Gebühren erhoben. Die Importeure rufen daher die Ware erst bei Bedarf ab. Damit läßt sich die Zahlung der Abgaben hinauszögern; die eigenen Lager bleiben entlastet. Nicht wenige Zollbeamte sind nebenberuflich als Berater für Importfirmen tätig. Dabei können Meinungsverschiedenheiten mit den Behörden auf informellem Wege, häufig allerdings zum Nachteil der öffentlichen Hand, ausgeräumt werden. Die Importeure sind deshalb auf die gesetzlich vorgesehenen Rechtsbehelfe und Rechtsmittel kaum angewiesen. Soweit der Betroffene dennoch gegen zollrechtliche Klassifikationsentscheidungen Widerspruch beim Comité Arancelario einlegt, ist im allgemeinen ein günstiger Verfahrensausgang zu erwarten; dafür dürften schon die in dem Gremium mitwirkenden Vertreter der Industrie- und Handelskammern Sorge tragen.

\section{Grundsteuer ${ }^{80}$}

Die Steuer wird in den meisten Fällen mit Hilfe der über jedes Grundstück geführten Steuerrolle erhoben. Dabei handelt es sich jedoch nicht um ein Steuerkataster, sondern um ein bloßes Eigentümerverzeichnis. Die darin enthaltenen Wertangaben, die auf jährlich vorzunehmenden Selbsteinschätzungen beruhen, sind viel zu niedrig. Eine realistische Erfassung der Werte scheitert auch daran, daß zahlreiche Grundeigentümer der gesetzlichen Verpflichtung zur Vorlage einer Steuererklärung nicht nachkommen. Von der Möglichkeit amtlicher Schätzung macht die Verwaltung seit einigen Jahren kaum noch Gebrauch. Sie sieht sich außerstande, den gerade gegen die Grundsteuer gerichteten Widerstand, insbesondere der einflußrei-

80 Der Gesetzestext selbst war bezeichnenderweise trotz intensiven Bemühens nicht aufzufinden, was angesichts der unzulänglichen Publikations- und Dokumentationsverhältnisse in diesem Lande nicht überraschen kann. Sogar bei den Beamten der Division Control del Impuesto Territorial war der Text nicht erhältlich. Daher muß auf Sekundärliteratur (Entwicklungsplan 1971-75, Anexo I, Tomo II, Cap. IX; Organización de Estados Americanos/Banco Interamericano de Desarrollo; Sistemas Tributarios de América Latina, Guatemala, Washington 1964, S. 27-31) sowie auf Auskünfte von Experten und Beamten der Steuerverwaltung zurückgegriffen werden. 
chen Großgrundbesitzer, zu brechen. Die in den Jahren 1967 und 1968 unternommenen Versuche, im Rahmen einer großangelegten Aktion durch staatliche Schätzer Neubewertungen vornehmen zu lassen - sie führte bei 36216 erfaßten Grundstücken zu einem Wertzuwachs von 190 Prozent (Q 69,9 Mio. gegenüber Q 24,1 Mio.) - wurden auf politischen Druck hin eingestellt.

In einigen Teilen des Landes wird die Wertbemessung anhand des in Aufbau befindlichen Steuerkatasters vorgenommen, das inzwischen die meisten Liegenschaften an der südlichen Pazifikküste und in der Hauptstadt erfaßt. Die Gebiete, in denen die Grundbesitzer von jeher dominieren und in denen die stärksten sozialen Gegensätze bestehen, blieben bis heute ausgespart.

Die Steuer ist in vierteljährlichen Raten zu entrichten. Zahlungsaufforderungen ergehen nicht; Säumnis bleibt von Rechts wegen ungeahndet, sofern die Zahlung bis Jahresende nachgeholt wird. Der bis 1970 angelaufene Zahlungsrückstand von mehr als Q 5 Mio. überstieg sogar das Aufkommen des Jahres 1971 (Q 4,84 Mio.) ${ }^{81}$. Die schlechte Steuermoral wird durch unzulängliche Kontrollen erleichtert. Es fehlt an ausreichendem und geeignetem Verwaltungspersonal. Zwangseintreibung findet sehr selten statt. Das einzig wirksame Druckmittel besteht darin, daß der Steuerschuldner sein Grundstück nur dann veräußern kann, wenn er dem Grundbuchamt eine Bescheinigung der Steuerbehörde vorlegt, wonach er seine Steuerpflicht erfüllt hat.

Gegen Verwaltungsakte der Steuerbehörden gibt es die Möglichkeit des Widerspruchs und Anfechtungsklage. Informationen über die Anzahl der eingelegten Rechtsbehelfe und Klagen waren nicht zu ermitteln.

81 Entwicklungsplan 1971-1975, Anexo I, Tomo II, IV - 5.

82 Art. 70 Ley de lo Contencioso-Administrativo. 
tion. The motivation of the employees to contribute to a continuous strengthening and development of the firms is only small up to now, though they will be the owners of $50 \%$ of the capital of the firm in the future. As to the relations between owners and employees the struggle for higher wages and salaries, better social securities and stability of the labor market is still dominating. By an systematic training and teaching the competent public institutions are engaged with the improvement of the educational level and the comprehension of the employees for their new claims and duties.

The last two paragraphs are concerned with the requirements and the changes just discussed, which are necessary in order to improve the Comunidad Industrial. However, by some groups in Peru the reformed private sector industrial firm is regarded to be only a transitional stage leading to a self-managed property. Some conclusions are presented at the end of the paper. The main one states that the Comunidad Industrial in its present form is no feasible way neither for the full participation of all industrial employees nor for a redistribution of property in the industrial sector.

\section{Taxes and Tax administration in Costa Rica and Guatemala}

\section{By Hermann Avenarius and Jürgen H. WolfF}

Despite very complex tax systems, in practice, the income, sales, and import taxes plus excise tax in Costa Rica and petroleum tax in Guatemala account for the bulk of the total revenue: two thirds in Costa Rica, more than three quarters in Guatemala. Indirect taxation prevails; the tax load is on par with (Costa Rica) or below (Guatemala) the Latin American average. The tax systems are characterised by a lack of logics: so, a large number of duties just exists on paper (more so in Guatemala than in Costa Rica). There are many unjustified exemptions reflecting, e. g., the political strength of the landlords in Guatemala. Finally, despite progressive rates in Guatemala, a marked inelasticity of the tax revenue in relation to economic development must be observed. The administrative processes, in both countries, do not provide for any efficient control and cross-checking of the tax return sheets filled out by the taxpayers. Consequently, the State does not succeed in enforcing the tax laws in the measure to be desired. A special problem in Guatemala is the fact that, until now, no systematic codification of legal sources concerning taxes exists.

The organization of the tax administration in both countries is characterised by a marked degree of geographical and organisational centralisation. In Costa Rica as in Guatemala, the customs and internal revenue administrations are clearly demarcated within the Ministries of Finance. In Costa Rica, the size of the different departments charged with the collection of internal taxes are out of proportion with the revenue of the taxes. In Guatemala, the structure of the internal tax administration introduced in 1972 is complicated by combining functional and formal criteria.

Once more, the research underlying this paper has demonstrated that administrative problems of developing countries cannot be defined in technical terms alone: What is unsatisfactory from a purely technical point of view or when applying 
universal values may be - and in fact, often is - completely functional within an existing political and social context. Consequently, aid projects which are flawless in technical terms often fail because they do not consider the power structure of the political and economic system.

\section{Korea - The Constitutional Problems of a Divided State In Comparison with the Federal Republic of Germany}

\section{By Youn-Soo Kim}

From the beginning, the constitutional problems of the divided states of Korea and Germany have been different. While the division of Germany is a "perpetual reparation", the division of Korea was the result of a military action. Therefore, Korea still retains the necessary foundations for uniting the nation in the future i. e. Territory, People and Sovereignty remain undivided in the eyes of international law. On the other hand, Germany has totally lost these foundations because of the conditions set by the neighboring countries and by the superpowers. Thus, Germany now consists, according to international law, of two seperate sovereign nations.

Of course, the validity of German and Korean constitutions is limited to the "half-states". In Korea, this led to the North- and South-Korean dialogues and resulted in the North-South-Agreement of July 4th 1972, relating to the peaceful unification of Korea on the principle of a homogeneous nation. The first step after this agreement was the 1972 amendment of both Korean Constitutions. This first attempt was unsuccessful because of the internal problems of South Korea. Future success in the peaceful unification of Korea requires that both North and South Korea must first build Democracy in these countries.

\section{Community Development in Asia}

\section{By Udo ERnSt Simonis}

If professional conferences are to be regarded as an adequate indicator, community development (c. d.) should gain a new momentum in theory as well as in practice. Several international conferences on this subject have taken place recently; others are planned for next year. Its not difficult to believe that they have shown and will show more of the deficiencies of the c. d. concept than of its achievements.

According to a United Nations definition, community development is the process by which the efforts of the people themselves are united with those of governmental authorities to improve the economic, social, and cultural conditions of communities, to integrate these communities into the life of the nation and to enable them to contribute fully to national progress. Judging from the statements at one of these conferences, this UN term is still adhered to by most countries, especially the developing countries of Asia. The notion, however, of what c. d. actually should be about and the real contents and dimensions of the c. d. programmes differ widely from country to country. There are examples (and sometimes models) where the 